\title{
Theory and experiment on laser-enabled inner-valence Auger decay of rare-gas atoms
}

\author{
X. M. Tong,,${ }^{1,2}{ }^{*}$ P. Ranitovic,${ }^{3}$ C. W. Hogle, ${ }^{3}$ M. M. Murnane, ${ }^{3}$ H. C. Kapteyn, ${ }^{3}$ and N. Toshima ${ }^{1}$ \\ ${ }^{1}$ Institute of Materials Science, Graduate School of Pure and Applied Sciences, University \\ of Tsukuba, 1-1-1 Tennodai, Tsukuba, Ibaraki 305-8573, Japan \\ ${ }^{2}$ Center for Computational Sciences, University of Tsukuba, 1-1-1 Tennodai, Tsukuba, Ibaraki 305-8577, Japan \\ ${ }^{3}$ JILA and Department of Physics, University of Colorado and NIST, Boulder, Colorado 80309-0440, USA
}

(Received 5 May 2011; published 11 July 2011)

\begin{abstract}
In rare-gas atoms, an inner-valence shell $n s$ hole cannot be filled by Auger decay because of an energy deficiency. We show theoretically and experimentally that by adding a moderately intense infrared laser, Auger decay is possible with decay rates increasing dramatically for laser intensities $\geqslant 10^{13} \mathrm{~W} / \mathrm{cm}^{2}$. For Xe atoms, the simulated laser-enabled Auger decay yields are comparable with the experimental one, while for Ar atoms, the simulated ones are much smaller. We attribute the discrepancies to screening effects of the photoelectron. Laser-enabled Auger decay is of fundamental importance for understanding attosecond science, and is also important for experimental applications in ultrafast atomic, molecular, and materials dynamics using $\mathrm{x}$ rays. More importantly it may provide a way to control the Auger decay time and selectively break chemical bonds of molecules using a control infrared laser field.
\end{abstract}

DOI: 10.1103/PhysRevA.84.013405

PACS number(s): $32.80 . \mathrm{Hd}, 32.80 . \mathrm{Qk}, 42.65 . \mathrm{Re}$

\section{INTRODUCTION}

When an inner-shell vacancy is created in atoms or molecules, the inner-shell core hole can be filled by another electron either through radiative decay or Auger decay. The ejected electron in Auger decay encodes atomic or molecular information, which can be used to diagnose the constituents of materials. Due to rapid advances in technology, it has become possible to create an inner-shell core hole using attosecond extreme ultraviolet (XUV) pulses and to manipulate the photoelectron or Auger electron using an ultrafast infrared (IR) laser. In such a laser-assisted process, a system is simultaneously irradiated by attosecond XUV pulses and laser beams. Characteristic sidebands appear surrounding the photoemission and Auger peaks, corresponding to simultaneous absorption and emission of laser photons. The magnitude and shape of these sidebands change as a function of the time delay between the attosecond XUV and laser pulses, encoding information about the XUV pulse duration and underlying electron dynamics. In recent years, laser-assisted photoemission [1,2] and laser-assisted Auger decay have been used to characterize ultrafast XUV pulses, to study electron-electron correlations in atoms and molecules [3], and to measure core-hole lifetimes of atoms [4-10] or of adsorbates on surfaces [11]. However, in all of those studies, laser-assisted Auger decay represented a means by which an existing Auger decay channel could be modified and exploited.

In a recent experiment [12], short XUV and IR pulses were used to doubly ionize Ar in a time-resolved manner. A 42-eV XUV pulse was used to populate Rydberg states of $\mathrm{Ar}^{+}$, just below the double-ionization (DI) threshold. In the presence of a moderately intense IR pulse, several DI pathways were discussed: IR-induced DI of the XUV-created $\mathrm{Ar}^{+*}$ and $\mathrm{Ar}^{* *}$ states, and laser-enabled Auger decay (LEAD). The double ionization of Ar through the LEAD process occurs when the

*tong@ims.tsukuba.ac.jp
XUV photon creates a $3 s$ inner-valence hole in the presence of a moderately intense laser field. Without the IR field, the $3 s$ hole is filled by a $3 p$ electron, radiatively relaxing on a nanosecond time scale. In the presence of an IR field, the $3 s$ hole can be filled by a $3 p$ electron on a femtosecond time scale though the LEAD process, where a second $3 p$ electron is ejected into an $\mathrm{Ar}^{+*}$ Rydberg orbit, or is promoted into the double-ionization continuum. The LEAD double-ionization channel opens up if the laser intensities are high enough so that the atomic ions can absorb enough energy through multiphoton processes to bridge the gap between the DI threshold and the $3 p \rightarrow 3 s$ energy difference as depicted in Fig. 1. In the case of $\mathrm{Ar}$, this energy is $14.08 \mathrm{eV}$, which is smaller than the ionization potential of $\mathrm{Ar}$ atoms. It was shown that even a moderate intensity of $10^{13} \mathrm{~W} / \mathrm{cm}^{2}$ can doubly ionize $\mathrm{Ar}$ with a rate higher than the rate of the $3 p \rightarrow 3 s$ radiative decay.

In this paper we expand the theoretical calculations to several other rare-gas atoms and show interesting experimental data. We organize this paper as follows: first we derive a general theory to describe the LEAD process, then we calculate the intensity-dependent LEAD rates for $\mathrm{Xe}, \mathrm{Kr}, \mathrm{Ar}$, and $\mathrm{Ne}$ atoms, and finally we present the experimental single- and double-ionization yields of $\mathrm{Xe}$ and $\mathrm{Ar}$ atoms by a $36-\mathrm{eV}$ XUV source with a moderate IR laser as a function of the femtosecond and attosecond time delays between the two pulses. Some of the observations can be explained by the LEAD process.

\section{THEORETICAL METHODS}

Figure 1 illustrates the LEAD process. Assuming that the wave functions of the atomic ion where an inner-valence hole $(N-1$ electron system), the doubly ionized ion core $\left(N-2\right.$ electron system), and the Auger electron are $\Psi_{h}(N-$ 1), $\Psi_{c}(N-2)$, and $\psi_{a}$ with the corresponding Hamiltonians $H_{h}(N-1), H_{c}(N-2)$, and $h_{a}$, respectively, we write the time evolution of the Auger electron wave function as (atomic 


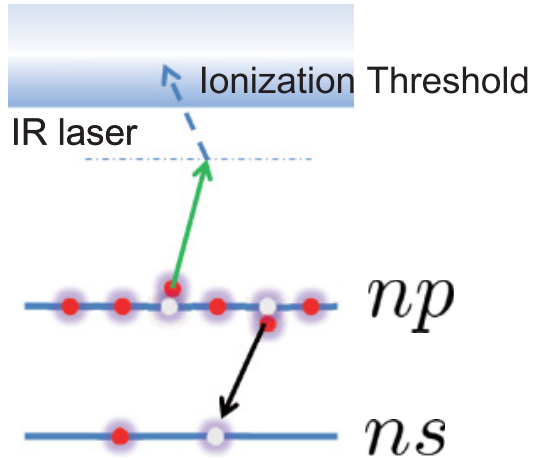

FIG. 1. (Color online) Schematic diagram of IR-enabled Auger decay in a rare-gas atom.

units $\hbar=m=e=1$ are used through the paper unless stated otherwise)

$$
\psi_{a}(\mathbf{r}, t)=-i \int_{-\infty}^{t} e^{-i \int_{\tau}^{t} h_{a}\left(t^{\prime}\right) d t^{\prime}} e^{-i\left(E_{h}-E_{c}\right) \tau} F(\mathbf{r}) d \tau,
$$

with

$$
F(\mathbf{r})=\left\langle\Psi_{c}(N-2)\left|V_{\text {res }}(\tau)\right| \Psi_{h}(N-1)\right\rangle,
$$

and $V_{\text {res }}(\tau)=H_{c}(N-2)+h_{a}(\tau)-H_{h}(N-1)$. Here $E_{h}$ and $E_{c}$ are the energies of the $\Psi_{h}(N-1)$ and $\Psi_{c}(N-2)$ states, respectively. To derive the above equation, we assume that the IR laser only affects the Auger electron having the occupied orbitals in $\Psi_{h}(N-1)$ and $\Psi_{c}(N-2)$ unperturbed. Since the Auger process is a two-electron process, the electron-laser field interaction does not appear in Eq. (2). Thus, $F(\mathbf{r})$ can be evaluated as

$$
F(\mathbf{r})=\sum_{i=1}^{N-2}\left\langle\Psi_{c}(N-2)\left|\frac{1}{\left|\mathbf{r}-\mathbf{r}_{i}\right|}\right| \Psi_{h}(N-1)\right\rangle .
$$

The Hamiltonian of the Auger electron in an IR field is written as

$$
h_{a}(t)=-\frac{\nabla^{2}}{2}+V_{\mathrm{eff}}(r)-z E(t),
$$

where $V_{\text {eff }}(r)$ is the model potential [13] of the atomic ions, $z$ the electron coordinate along the IR polarization direction, and $E(t)$ the IR field strength. For rare-gas atoms ( $\mathrm{Ne}, \mathrm{Ar}, \mathrm{Kr}$, or $\mathrm{Xe}),\left|\Psi_{h}(N-1)\right\rangle$ stands for $\left|n s n p^{6}{ }^{2} S\right\rangle$, which can be recast as [14]

$$
\begin{aligned}
\left|\Psi_{h}(N-1)\right\rangle= & \frac{1}{\sqrt{15}}\left|n s\left(n p^{4}{ }^{1} S\right) n p^{2}{ }^{2} S\right\rangle \\
& +\frac{1}{\sqrt{3}}\left|n s\left(n p^{4}{ }^{1} D\right) n p^{2}{ }^{2} S\right\rangle \\
& +\sqrt{\frac{3}{5}}\left|n s\left(n p^{4}{ }^{3} P\right) n p^{2}{ }^{2} S\right\rangle .
\end{aligned}
$$

$\left|\Psi_{c}(N-2)\right\rangle$ is $\left|n s^{2} n p^{4}{ }^{1} S\right\rangle$ or $\left|n s^{2} n p^{4}{ }^{1} D\right\rangle$ where $n$ is the principal quantum number of the highest occupied orbital. The last term in Eq. (5) does not contribute to the LEAD due to the conservation of angular momentum. The final doubly charged ions created by the LEAD can be in the ${ }^{1} S$ (the upper limit is $1 / 15$ ) or ${ }^{1} D$ (the upper limit is $1 / 3$ ) states, limiting the total double-ionization yield to be $40 \%$ of the hole population.
Defining a radial function $f(r)$ as

$$
f(r)=\int_{0}^{\infty} R_{n s}\left(r_{1}\right) \frac{r_{<}}{r_{>}^{2}} R_{n p}\left(r_{1}\right) R_{n p}(r) r_{1}^{2} d r_{1},
$$

where $r_{<}\left(r_{>}\right)$stands for the smaller (larger) one of the $r$ and $r_{1}$, and $R_{n s}$ and $R_{n p}$ are the radial wave functions of the $n s$ and $n p$ states, we obtain

$$
F(\mathbf{r})= \begin{cases}-\frac{1}{\sqrt{3}} f(r) Y_{00}(\hat{\mathbf{r}}) & \text { for }{ }^{1} S, \\ -\sqrt{\frac{2}{15}} f(r) Y_{2 M}(\hat{\mathbf{r}}) & \text { for }{ }^{1} D .\end{cases}
$$

${ }^{1} S$ ( $\left.{ }^{1} D\right)$ stands for the final state of the doubly ionized species. Using the expression for $F(\mathbf{r})$ and the second-order split-operator method, we calculated the Auger election wave function from Eq. (1) numerically using the generalized pseudospectral method in the energy representation [15]. The single-electron wave functions of the occupied orbitals in $\left|\Psi_{h}(N-1)\right\rangle$ and $\left|\Psi_{c}(N-2)\right\rangle$ are calculated from the density functional theory with the self-interaction-correction method [16].

To speed up the convergence of the simulation, we added a temporal window function $e^{-\tau^{2} / T^{2}}$ in Eq. (1). Thus, after a long propagation time we can expand $\psi_{a}$ as

$$
\psi_{a}(\mathbf{r}, t \rightarrow \infty)=\int C(\epsilon) \psi_{\epsilon}(\mathbf{r}) d \epsilon,
$$

where $\psi_{\epsilon}(\mathbf{r})$ is the laser-field-free atomic continuum wave function, and $\epsilon$ is the Auger electron energy. The energy distribution of the emitted electron is expressed as

$$
\frac{d P(\epsilon)}{d \epsilon}=|C(\epsilon)|^{2},
$$

and the LEAD rate is written as

$$
R_{a}=\frac{\sqrt{2 \pi}}{T} \int_{0}^{\infty} \frac{d P(\epsilon)}{d \epsilon} d \epsilon
$$

The $\sqrt{2 \pi} / T$ term is introduced because we added a temporal window function. If there is no IR laser field, Eq. (10) reduces to the standard expression for the Auger decay rate. Due to the energy deficiency, the Auger decay rate goes to zero for a valence $n s$ hole of rare-gas atoms without the IR field. The numerical simulations of the LEAD process are similar to the simulations of the above-threshold ionization (ATI) spectra, and the details can be found in [17,18].

\section{THEORETICAL RESULTS AND DISCUSSION}

Based on the above method, we calculate the LEAD rates of $\mathrm{Xe}, \mathrm{Kr}$, Ar, and Ne rare-gas atoms. In the simulation, we choose an IR pulse with a 40-fs pulse duration [the full width at half maximum (FWHM)], wavelength of $800 \mathrm{~nm}$, and a moderate laser intensity to avoid direct laser ionization of the outer shell electron. The numerical convergence was tested by varying the number of grid points, partial waves, and sphere size. The numerical data presented here were calculated with a sphere radial size of 200 a.u. (a.u. denotes atomic units), 512 grid points, and 32 partial waves. 

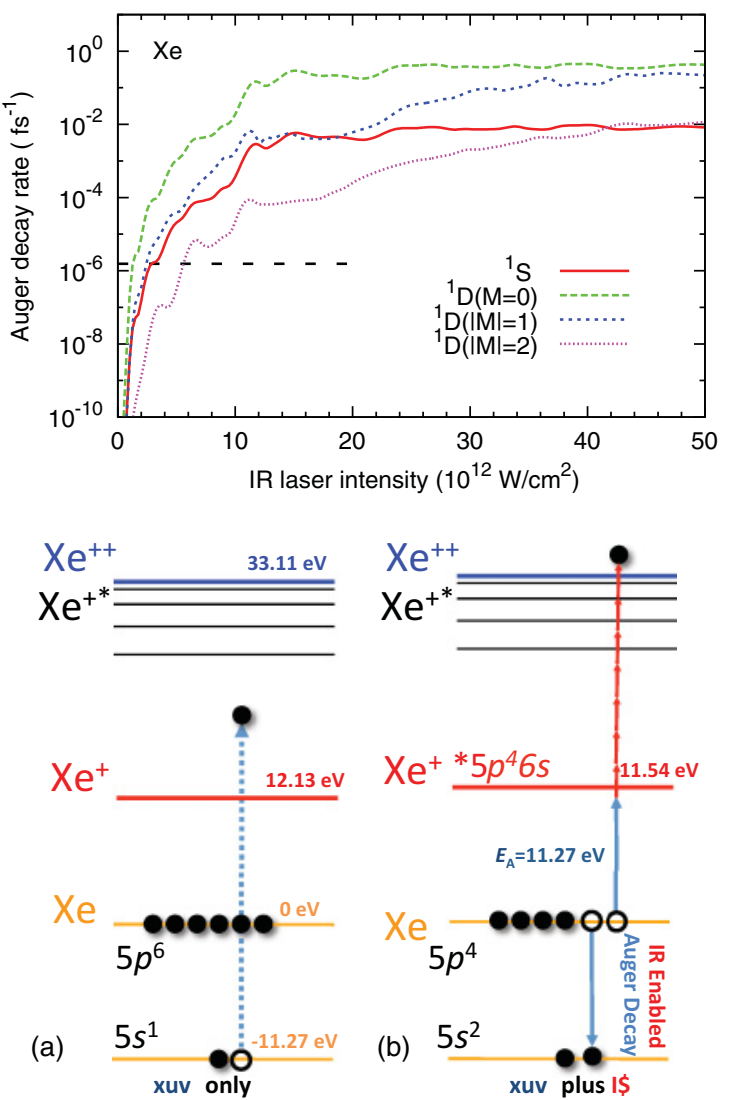

FIG. 2. (Color online) Upper panel: IR-enabled Auger decay rates for Xe, with $\mathrm{Xe}^{2+}$ ions in the ${ }^{1} S$ state (solid line) and ${ }^{1} D$ states (dashed lines). The horizontal dashed line indicates the radiative decay rate of the hole. Lower panel: (a) Xe energy diagram for the lowest singleand double-ionization thresholds with a $5 s$ electron ionized by an XUV pulse. (b) A $5 p$ electron can be ejected to an $\mathrm{Xe}^{+*}$ Rydberg orbit or the $\mathrm{Xe}^{++}$continuum through the LEAD process after a respective absorption of at least one or seven IR photons.

Figure 2 (upper panel) shows the LEAD rates of Xe as a function of the IR laser intensity for the doubly ionized ionic core in the ${ }^{1} S$ and ${ }^{1} D$ states.

To check how the LEAD rates change as a function of the propagation time, we also calculated the decay rates of the ${ }^{1} D$ state with $M=0$, for an 80-fs FWHM temporal function. The decay rates do not depend on the propagation time. At lower IR laser intensities $\left(<10^{12} \mathrm{~W} / \mathrm{cm}^{2}\right)$, the LEAD rates are negligibly small. The rates increase dramatically when the IR intensity increases to $4 I_{0}$ with $I_{0}=10^{12} \mathrm{~W} / \mathrm{cm}^{2}$. For $M=0$ states, the rates reach peak values for the laser intensity of $11 I_{0}$, and then decrease slowly. By analyzing the energy structures of Xe ions with a $5 s$ hole as shown in Fig. 2 (lower panel), we find that the $6 s$ orbital energy is close to the energy difference of $E_{h}$ and $E_{c}$. Thus, in the presence of the IR laser field, the $5 s$ hole can be filled by one of the $5 p$ electrons, while another $5 p$ electron is excited to the $\mathrm{Xe}^{+*}$ Rydberg states temporally. Figure 2 (lower panel) shows that the $6 s$ orbital is the lowest possible Rydberg state, and it can be easily ionized by the IR laser with the absorption of at least seven IR photons.

Note that the LEAD rate depends on the energy defect, and that the IR intensity also increases this defect through

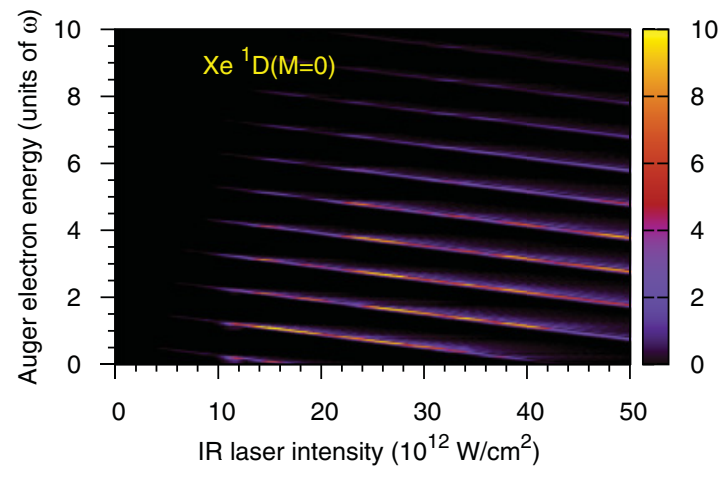

FIG. 3. (Color online) Energy distribution of the Auger electron as a function of the IR laser intensity for $\mathrm{Xe}$, with the $\mathrm{Xe}^{2+}$ ions in the ${ }^{1} D(M=0)$ state.

the ponderomotive shift of the atomic energy levels $[19,20]$. Thus, as the IR laser intensity increases, the LEAD rates may decrease slowly, as shown in Fig. 2 (upper panel). This ponderomotive effect can be seen more clearly in the energy spectra of the Auger electrons as shown in Fig. 3. The Auger electron spectra are similar to the ATI spectra of atoms in a laser field [21-23], with sharp peaks separated by one IR photon energy. Below the IR laser intensity of $20 I_{0}$, the IR laser can doubly ionize Xe by adding at least seven IR photons to the Auger $5 p$ electron. As the intensity increases above $20 I_{0}$, the IR laser can doubly ionize Xe by adding at least eight IR photons to the Auger electron, thus decreasing the decay rates.

LEAD is a general laser-enabled process and it should also occur in other rare-gas atoms. Figures 4 shows the LEAD rates for $\mathrm{Kr}$, Ar, and Ne. Different from the Xe case, we present the LEAD rates for the ${ }^{1} S$ and ${ }^{1} D$ states without the detail distribution of the $M$ sublevels. In these gases, the general trend of the LEAD rates as a function of the IR laser intensity is similar to that in $\mathrm{Xe}$. For $\mathrm{Kr}$ and $\mathrm{Ar}$, the leading decay channel is the ${ }^{1} D$ state, similar to $\mathrm{Xe}$. We see that the relative contribution of the ${ }^{1} S$ state increases in the $\mathrm{Kr}$ and Ar targets. On the other hand, the leading decay channel in $\mathrm{Ne}$ is ${ }^{1} S$, while the contribution from the ${ }^{1} D$ channel is much smaller.

These results can be explained in terms of the relevant atomic energy structures. The energy defects of the $n s$ hole to the double-ionization threshold calculated by the method described in Ref. [16] are 18.56, 14.08, 10.90, and $9.80 \mathrm{eV}$ for $\mathrm{Ne}, \mathrm{Ar}, \mathrm{Kr}$, and $\mathrm{Xe}$, respectively. The IR laser field can easily induce the Auger decay for a small energy defect, namely, for $\mathrm{Xe}$ and $\mathrm{Kr}$. This explains why we need a high IR laser intensity to induce the LEAD process in the case of Ne.

To explain the differences in the contributions of the final doubly charged states for different targets, we intuitively analyze the overlap of the outgoing partial waves and the relevant electronic structures of $\mathrm{Ne}, \mathrm{Ar}, \mathrm{Kr}$, and $\mathrm{Xe}$. The precursor state $(3 d)$ of a $d$ partial wave has a very small overlap with the inner-valence $2 s$ and $2 p$ orbitals in $\mathrm{Ne}$, and therefore the contribution from the $s$-channel is dominant. In the case of $\mathrm{Xe}$, the $4 d$ orbital is an occupied orbital and the overlap of the $d$ states with the $5 s$ and $5 p$ orbitals is larger than the overlap of the $s$ states. This results in a larger contribution from the ${ }^{1} \mathrm{D}$ states, compared with the $\mathrm{Kr}$ case. For Ar, the $3 d$ orbital 

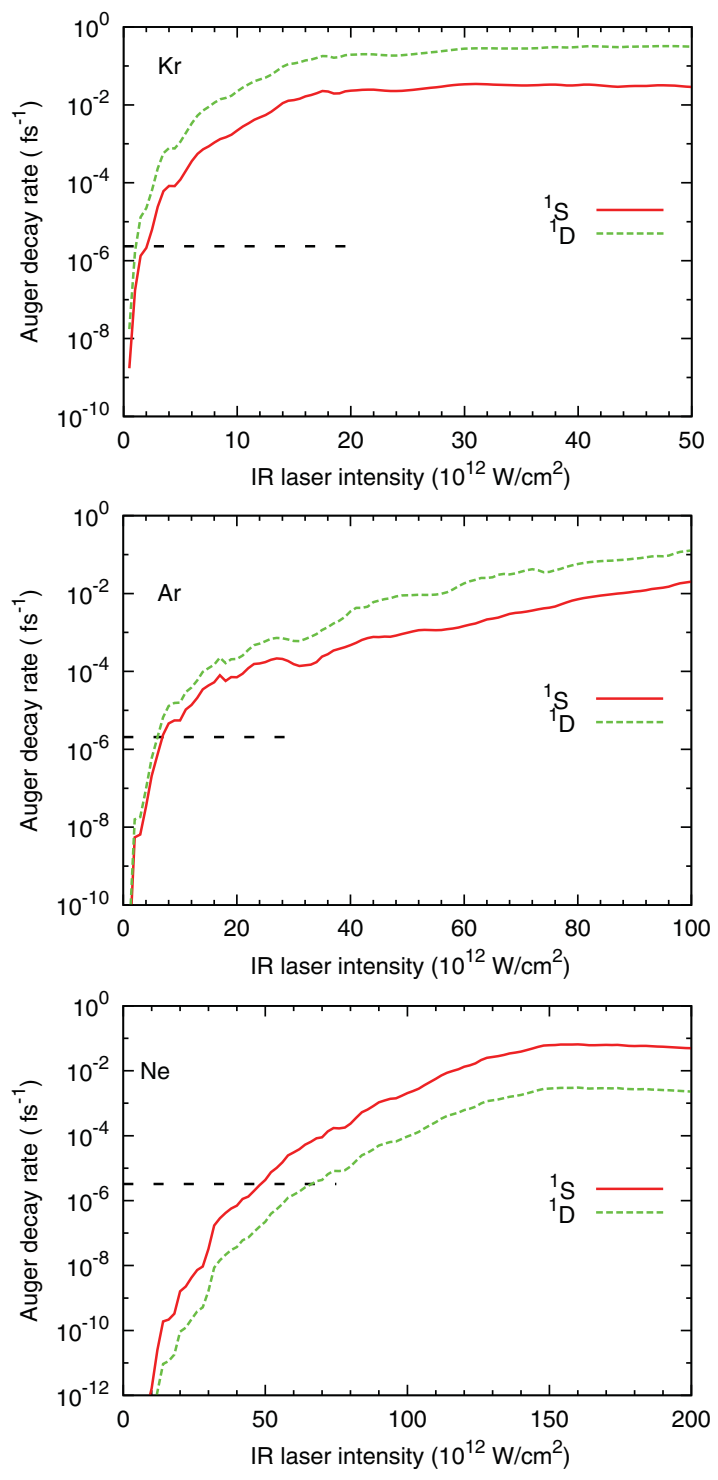

FIG. 4. (Color online) IR-enabled Auger decay rates for $\mathrm{Kr}$, Ar, and Ne to ${ }^{1} S$ and ${ }^{1} D$. The horizontal dashed lines indicate the radiative decay rates of the holes.

is not occupied but has a large overlap with the inner-valence orbitals $3 s$ and $3 p$. This nicely explains why the $d$ channel contributes more than the $s$ channel for $\mathrm{Ar}, \mathrm{Kr}$, and $\mathrm{Xe}$.

\section{EXPERIMENTAL RESULTS AND DISCUSSION}

To find evidence of LEAD processes, we performed experiments on the double ionization of $\mathrm{Ar}$ and $\mathrm{Xe}$ atoms using time-resolved femtosecond IR and 36-eV XUV pulses in a cold target recoil ion momentum spectroscopy (COLTRIMS) geometry. For Ar, $36 \mathrm{eV}$ is well below the double-ionization threshold, and several possible DI channels open up in the presence of an IR pulse as intense as $7 \times 10^{12} \mathrm{~W} / \mathrm{cm}^{2}$ [12]. For $\mathrm{Xe}$, the $36 \mathrm{eV}$ photon energy is just above the DI threshold and the number of DI channels significantly reduces compared with the $\mathrm{Ar}$ case. For example, $\mathrm{Xe}^{* *}\left(5 p^{4}\right)$ and $\mathrm{Xe}^{+*}\left(5 p^{5}\right)$ states are not possible, as was the case in Ar. In Xe, a $36 \mathrm{eV}$ XUV photon can create a $5 s$ hole and leave the singly charged Xe in the ground state after a $5 p-5 s$ radiative relaxation, or leave $5 s$ $\mathrm{Xe}$ in a highly excited state [Fig. 2 (lower panel)]. In the former case, the IR pulse can ionize $\mathrm{Xe}^{+}$through the LEAD process with rates several orders of magnitude larger than the radiative $5 p-5 s$ decay [Fig. 2 (upper panel)]. The ability to enable Auger decay with rates significantly higher than radiative decay opens up possibilities for controlling electron-electron interactions in atoms and molecules.

Our experimental setup consists of a high-power $(30 \mathrm{~W})$, high repetition rate $(10 \mathrm{kHz}) 30-\mathrm{fs} \mathrm{Ti}$ :sapphire laser system $(785 \mathrm{~nm})$, a gas-filled waveguide for generating harmonics, and a COLTRIMS apparatus which allows the simultaneous detection of ion and electron three-dimensional momenta. Using part of the laser output, high harmonics are generated in Ar gas and then refocused into separate Ar and Xe supersonic gas jets using a pair of multilayer XUV mirrors which reflect photon energies around $36 \mathrm{eV}$. A 200-nm thick Al filter is inserted in the XUV beam to attenuate the driving IR pulses reflected from the XUV mirrors. Even a weak IR laser beam copropagating with the XUV beam can damage the XUV coating. This results in higher reflectivity of lower harmonics. In this case, the 13th and 15th harmonics are reflected from the XUV optics as seen in the Ar photoelectron spectrum shown in Fig. 5 (lower panel). Another part of the laser beam is temporally and spatially recombined with the XUV beam in a collinear fashion, and focused onto the Xe and Ar targets at a laser intensity of $7 \times 10^{12} \mathrm{~W} / \mathrm{cm}^{2}$. A linear stage, with 260 -as resolution, was used to delay the IR pulse relative to the XUV pulse. The XUV pulse duration was approximately $10 \mathrm{fs}$.

Figure 5, upper and lower panels, respectively, shows the Ar DI yield as well as the ratios of $\mathrm{Ar}^{++} / \mathrm{Ar}^{+}$yields, and a photoelectron yield, taken in coincidence with $\mathrm{Ar}^{+}$atoms,
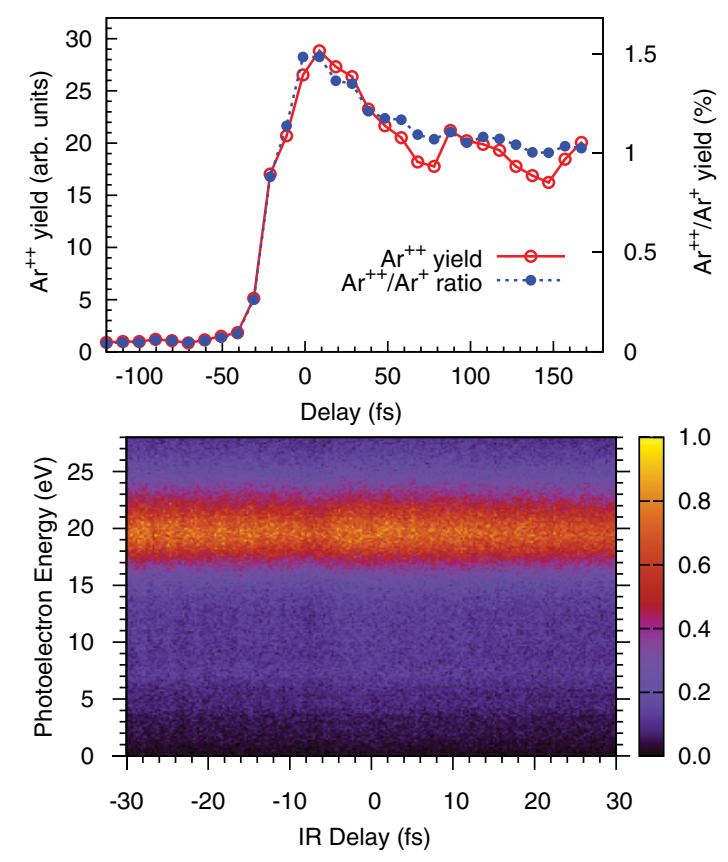

FIG. 5. (Color online) $\mathrm{Ar}^{++}$and $\mathrm{Ar}^{++} / \mathrm{Ar}^{+}$yields as a function of the delay between the XUV and IR pulses (upper panel). The photoelectron spectra taken in coincidence with $\mathrm{Ar}^{+}$ions in the overlap region (lower panel). 
as a function of the XUV-IR relative delay. Negative delays represent the case when the IR pulse precedes the XUV pulse, while positive delays present the case when the IR pulse arrives after the XUV pulse. The two pulses overlap temporally for the delays between -30 and +30 fs (overlap region). Figure 5, upper panel, shows that the moderate IR field enhances the $\mathrm{Ar}^{++}$yield by roughly 30 times in the overlap region, while the $\mathrm{Ar}^{++} / \mathrm{Ar}^{+}$ratio maximizes at about $1.6 \%$. The photoelectron yield taken in coincidence with $\mathrm{Ar}^{+}$ions (Fig. 5, lower panel) shows a peak around $20 \mathrm{eV}$ corresponding to photoionization of a $3 p$ electron by the $36-\mathrm{eV}$ XUV photon. If the XUV pulse ionizes a $3 s$ electron (binding energy $29.2 \mathrm{eV}$ ), we expect to see a photoelectron signal around $6.2 \mathrm{eV}$, after subtracting the ponderomotive potential of about $500 \mathrm{meV}$. The photoelectron yield in that region shows two lines at 4.2 and $7.4 \mathrm{eV}$, corresponding to a $3 p$ electron ionized by the 13th and 15th harmonics "leaking" though the XUV reflective optics. Since the $36-\mathrm{eV}$ XUV photon is $7.5 \mathrm{eV}$ below the double-ionization threshold, the $\mathrm{Ar}^{++}$enhancement in the overlap region could come from coupling of the $\mathrm{Ar}^{+*}$ and $\mathrm{Ar}^{* *} 3 p$ states into the double-ionization continuum by the IR pulse. Single-photon double ionization is a resonant process and the $\mathrm{Ar}^{++}$yield of IR field ionization of $\mathrm{Ar}^{* *}$ should be sensitive to the XUV energy. The pattern of the $\mathrm{Ar}^{++} / \mathrm{Ar}^{+}$ yield obtained by the $36-\mathrm{eV}$ XUV photon is similar to the one obtained by the 42-eV XUV source [12]. Therefore, the additional DI yield enhancement also comes from a LEAD process where the $36-\mathrm{eV}$ photon ionizes a $3 s$ electron, while the IR field couples an Auger $3 p$ electron to the DI continuum.

Figure 6, upper and lower panels, respectively, shows a $\mathrm{Xe}$ double-ionization yield as well as the ratios of $\mathrm{Xe}^{++} / \mathrm{Xe}^{+}$ yields, and the photoelectron yield, as a function of XUV-IR relative delay. The data was taken by using similar XUV and IR parameters as for the Ar data shown in Fig. 5. By doubly ionizing Xe using a $36-\mathrm{eV}$ XUV pulse, we make sure that the highest double-ionization threshold $\left({ }^{1} D_{2}\right.$ $35.5 \mathrm{eV}$ ) is still below the XUV photon energy. By doing so, we eliminate the DI contribution from the $\mathrm{Xe}^{+*} / \mathrm{Xe}^{* *} 5 p$ states ionized by the IR pulse. This results in a significantly smaller enhancement of the $\mathrm{Xe}^{++}$yield in the overlap region, compared with the $\mathrm{Ar}^{++}$enhancement (30:1.7 in favor of $\mathrm{Ar}$ ), and in a higher $\mathrm{Xe}^{++} / \mathrm{Xe}^{+}$ratio (4:1.6 in favor of $\mathrm{Xe}$ ). The flat $\mathrm{Xe}^{++}$yield for positive IR delays shows that short-lived, autoionizing double excited states probably do not play a significant role in this case. From the photoelectron spectra [Fig. 6 (lower panel)], we notice that the lower harmonics (13th and 15th) contribute more significantly to the $\mathrm{Xe}^{+}$ yield compared with the Ar case. Thus, the $\mathrm{Xe}^{++} / \mathrm{Xe}^{+}$yield coming from the $36-\mathrm{eV}$ photon channel is probably even higher than shown in Fig. 6 (upper panel). In the case of Xe, $7 \times$ $10^{12} \mathrm{~W} / \mathrm{cm}^{2}$ IR intensity can ionize $\mathrm{Xe}$, as seen at $0.2-\mathrm{eV}$ electron energy at both positive and negative delays in Fig. 6. By setting up the experiment in this way, we make sure that the LEAD process is experimentally possible, since the Xe LEAD deficiency is only $9.80 \mathrm{eV}$. However, even though we were able to decrease the number of the IR-enabled double-ionization channels by using an XUV photon energy just above the DI threshold, isolating unambiguously the LEAD process remains an experimental challenge.
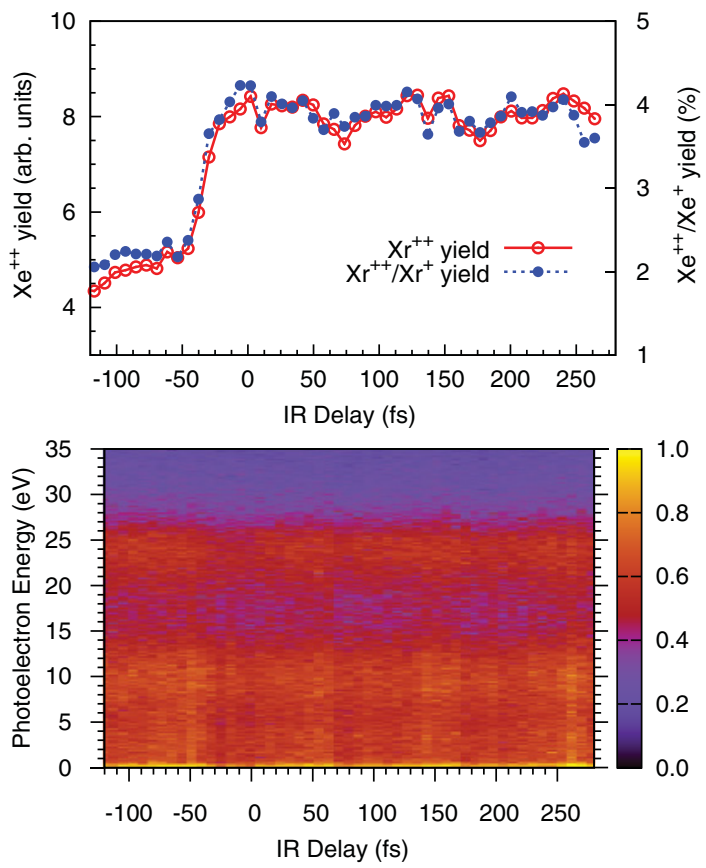

FIG. 6. (Color online) $\mathrm{Xe}^{++}$and $\mathrm{Xe}^{++} / \mathrm{Xe}^{+}$yields as a function of the delay between the XUV and IR pulses (upper panel). Photoelectron spectra taken simultaneously with the Xe ions (lower panel).

In addition to exploring femtosecond delays, we performed a time-resolved experiment with attosecond resolution in the overlap region. Figure 7 shows the $\mathrm{Xe}^{++}$versus $\mathrm{Xe}^{+}$yield as a function of the XUV-IR delays. In this experiment, in addition to the 36-eV XUV harmonic we allow the lower harmonics (lower than the Xe ionization potential) to populate neutral $\mathrm{Xe}^{*}$ states. We did this by taking the Al filter out of the XUV beam. By doing this, we lock a weak IR pulse $(3 \times$ $10^{11} \mathrm{~W} / \mathrm{cm}^{2}$ ) to the XUV pulse in the time domain, and let both beams copropagate. The inset of Fig. 7 shows the XUV and IR

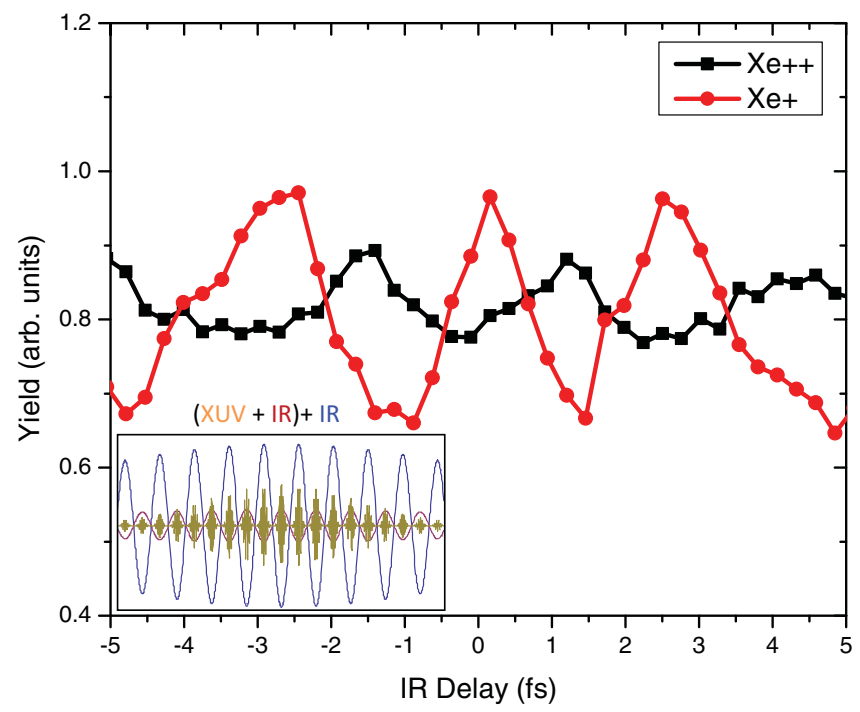

FIG. 7. (Color online) $\mathrm{Xe}^{+}$and $\mathrm{Xe}^{++}$yields as a function of the XUV-IR pump and IR probe pulses. The XUV and IR pulses in the time domain are shown in the inset. 
pulses in the time domain. As we delay the probe IR beam $(7 \times$ $10^{12} \mathrm{~W} / \mathrm{cm}^{2}$ ) on an attosecond time scale relative to the XUVIR pump beam, we change the total intensity the atom sees at the instant of the strobe XUV pulse [24,25]. By doing so we control the $\mathrm{Xe}^{++} / \mathrm{Xe}^{+}$yields both by modulating the total IR intensity and by interfering the electron wave packets. The phase difference of about a half optical cycle is telling us that different processes are responsible for the coherent control of the single- and double-ionization Xe yields. The interference of the two electronic wave packets with the final energy of the 8th harmonic (7th+IR and 9th-IR) are responsible for the modulation of the $\mathrm{Xe}^{+}$yield, while the modulation of the $\mathrm{Xe}^{++}$ yield can occur through several different channels. The LEAD channel is very sensitive to the IR intensity as shown in Fig. 2, but also it is possible that the phase of the XUV and IR pulses plays a role. Thus, we are not surprised that the $\mathrm{Xe}^{+}$and $\mathrm{Xe}^{++}$ yields get modulated at different phases, and with different amplitudes. To compare our calculations with this experiment and to gain insight into the physical processes, we need to convolve the time-dependent Auger electron wave function in Eq. (1) with the time-dependent photoelectron wave function of the $n s$ orbital ionized by the XUV pulse in the presence of the IR field. In this way we can retain the history of the creation of the $n s$ hole and also the phase information. We expect that the LEAD process would depend on the time delay between the attosecond XUV pulse (creation of the hole) and the probing IR field (induced Auger decay). Thus we can both induce Auger decay by employing an IR laser field and control the process by tuning the time delay between the two pulses.

\section{SCREENING EFFECTS}

Comparing the calculated LEAD rates with the experimental observation for Xe atoms, we found that the decay rates are on the order of $10^{-2} \mathrm{fs}^{-1}$ at the applied IR intensity. Therefore, once a $5 s$ hole is created, it can easily decay through the LEAD process when the IR laser is applied. For Ar atoms, the LEAD rates are on the order of $10^{-5} \mathrm{fs}^{-1}$ at the applied IR intensity. So the contribution from the LEAD should be negligible. This contradicts the LEAD explanation of our experimental observations. Where is the problem?

So far, we focused our attention on the general phenomena of laser-enabled Auger decay, assuming that the subvalence $n s$ hole has been created before applying the IR field and that the $n s$ hole has no phase relation with the probe (enabling) IR laser. We have also ignored the screening effect due to the spectator photoelectron, which in general reduces the energy defect. If the XUV and IR pulses arrive simultaneously, the photoelectron is still in the vicinity of the parent ionic core when the IR-enabled Auger decay happens. The presence of the spectator photoelectron reduces the energy defect, which increases the LEAD rate significantly. The outerscreening effect can be taken into account by replacing the electron-nuclear Coulomb interaction with a Debye screening potential as

$$
V_{c}(r)=-\frac{Z}{r} \rightarrow V(r)=-\frac{Z}{r} e^{-r / \lambda_{d}},
$$

where $Z$ is the atomic number and $\lambda_{d}$ is the Debye length. Using the density functional theory with the

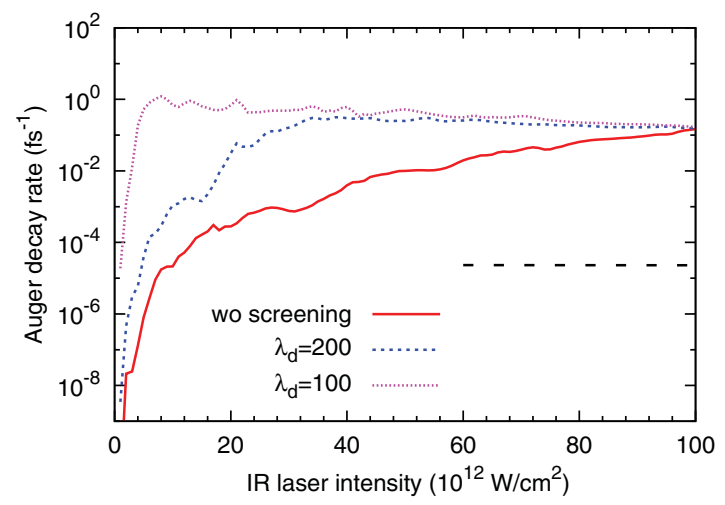

FIG. 8. (Color online) Total IR-enabled Auger decay rates of $\mathrm{Ar}$ atoms for different Debye lengths.

self-interaction-correction method [15], we obtain the energy defect and the effective potential. With the effective potential, we can calculate the orbital wave functions and the LEAD rates. The Debye length can be estimated by replacing the temperature with the kinetic energy of the photoelectron and by using the density as $3(v t)^{-3} / 4 \pi$ with $v$ the velocity of the photoelectron and $t$ the time after the creation of the hole. With these assumptions, the Debye length can be expressed as

$$
\lambda_{d}=\sqrt{3 v^{5} t^{3} / 2} .
$$

From the present experiment conditions, Debye screening effect lasts for an order of $10 \mathrm{fs}$.

Taking the Ar atom as an example, the energy defect reduces from $14.1 \mathrm{eV}$ (without screening) to 13.2 (for $\lambda_{d}=500 \mathrm{a} . \mathrm{u}$.), 11.8 (for $\lambda_{d}=200$ a.u.), and $9.4 \mathrm{eV}$ (for $\lambda_{d}=100$ a.u.). The total LEAD rates with the screening effect are plotted in Fig. 8. Overall, we see that the screening effect increases the LEAD rates greatly for the lower IR intensities, and the rates reach saturation quickly for $\lambda_{d}=100$ a.u., corresponding to a strong screening case. For a weak screening case, $\lambda_{d}=200$ a.u., the LEAD rate decreases. This explains why the DI yield maximizes in the overlap region between the IR and XUV pulses then decreases as the time delay increases for Ar. For $\mathrm{Xe}$, the additional yield enhancement in the overlap region is absent. At the IR laser intensity used in the experiment, we expect the LEAD Xe ${ }^{++}$yield enhancement to be saturated due to the smaller energy deficiency, thus making no difference whether the XUV and IR pulses are temporally overlapped, or the IR pulse arrives after the XUV pulse.

As we discussed above, LEAD processes can be further classified into two types: a resonant LEAD process in which there is one dominant bound state involved as an intermediate state and a nonresonant LEAD process in which there is no such dominant bound state. For the resonant LEAD process, the LEAD rate strongly depends on the energy structure of the atomic ions or the model potential used in the simulation. Figure 9 shows the total LEAD rates calculated using two different model potentials. One is a six-parameter model potential [13] and the other is a three-parameter potential [26] as $V(r)=$ $-\left[2+a e^{-b r}+(16-a) e^{-c r}\right] / r$ with $a=8.9, b=1.5, c=$ 6.1. The parameters are obtained by fitting the numerical potential of $\mathrm{Ar}^{+}$from density functional theory [16]. The general 


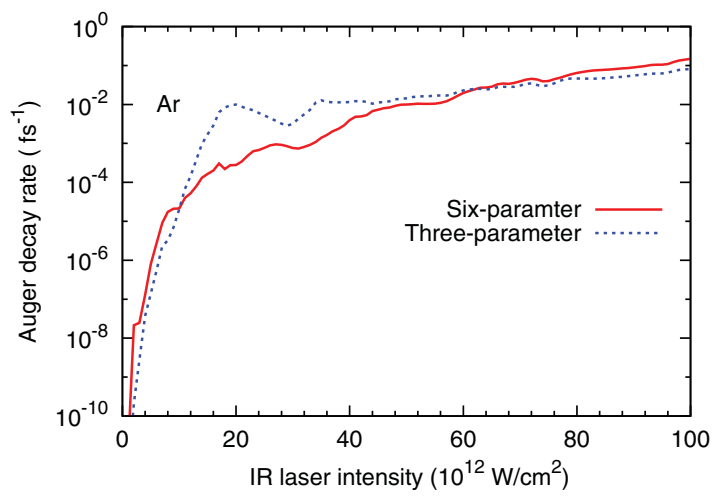

FIG. 9. (Color online) Total IR-enabled Auger decay rates of Ar atoms calculated from different model potentials.

trends of the LEAD rates obtained from the two model potentials are close to each other. A large discrepancy appears at $2 \times$ $10^{13} \mathrm{~W} / \mathrm{cm}^{2}$. By analyzing the energy spectra of the Auger electron, we attribute the discrepancy to a resonant LEAD process. The quantitative differences predicted by different model potentials do not change the physical conclusion of this study.

Note that a similar Xe experiment was reported by Guyetand et al. [27]. The data shown there, even though lacking in statistics, are an example of a state-of-the-art COLTRIMS experiment, and already showed the LEAD double-ionization channel. From Fig. 2, it is obvious that LEAD double ionization would produce two electrons that share the energy unequally. The first electron is ionized from the $5 s$ subshell by the XUV photon and carries a discreet energy, while the second electron emerges with low kinetic energies in the form of ATI peaks (Fig. 3). In this case even the electron angular distributions are different. The XUV electron angular distribution should have signature of a $p$ partial wave, while the second, a LEAD $p$ electron absorbs seven additional IR photons. The authors observed this channel and noticed the nonlinear sharing (region 4 in Fig. 14 of Ref. [27]) but did not consider a possibility of explaining the channel through LEAD process.

\section{SUMMARY}

We developed a general theoretical method for calculating LEAD yields and applied it to rare-gas atoms. We showed that the presence of a moderate IR field can turn on an Auger decay of an $n s$ subvalence shell with rates several orders of magnitude higher than the radiative decay through which an atom naturally relaxes without the presence of an IR laser field. We discussed how screening of an outgoing $n s$ electron can increase the LEAD yield when the XUV and IR pulses overlap in time. By doing two-color XUV-IR attosecond and femtosecond time-resolved experiments in $\mathrm{Xe}$ and $\mathrm{Ar}$, we separated several different IR-induced double-ionization channels and discussed ways of controlling Auger decay processes. In future work, we will focus on the coherent control processes and attosecond electron-electron dynamics in atomic and molecular targets.

\section{ACKNOWLEDGMENTS}

This research was supported by a Grand-in-Aid for Scientific Research (C), from the Japan Society for the Promotion of Science, and by the U.S. National Science Foundation. We thank Achim Czasch, Till Jahnke, and RoentDek for the COLTRIMS support, and Yanwei Liu, Farhad Salmassi, and Eric Gullikson of LBNL for their help with the XUV mirror coatings.
[1] T. E. Glover, R. W. Schoenlein, A. H. Chin, and C. V. Shank, Phys. Rev. Lett. 76, 2468 (1996).

[2] L. Miaja-Avila, C. Lei, M. Aeschlimann, J. L. Gland, M. M. Murnane, H. C. Kapteyn, and G. Saathoff, Phys. Rev. Lett. 97, 113604 (2006).

[3] H. Aksela and S. Aksela, Rev. Phys. Chem. 76, 370 (2007).

[4] M. Drescher, M. Hentschel, R. Kienberger, M. Uiberacker, V. Yakovlev, A. Scrinizi, T. Westerwalbesloh, U. Kleineberg, U. Heinzmann, and F. Krausz, Nature (London) 419, 803 (2002).

[5] T. Mercouris, Y. Komninos, and C. A. Nicolaides, Phys. Rev. A 76, 033417 (2007).

[6] C.-H. Zhang and U. Thumm, Phys. Rev. A 80, 032902 (2009).

[7] C. Buth and K. J. Schafer, Phys. Rev. A 80, 033410 (2009).

[8] A. K. Kazansky, I. P. Sazhina, and N. M. Kabachnik, J. Phys. B 42, 245601 (2009).

[9] Y.-C. Chiang, P. V. Demekhin, A. I. Kuleff, S. Scheit, and L. S. Cederbaum, Phys. Rev. A 81, 032511 (2010).

[10] T. Shimizu, T. Sekikawa, T. Kanai, S. Watanabe, and M. Itoh, Phys. Rev. Lett. 91, 017401 (2003).
[11] L. Miaja-Avila, G. Saathoff, S. Mathias, J. Yin, C. La-o-vorakiat, M. Bauer, M. Aeschlimann, M. M. Murnane, and H. C. Kapteyn, Phys. Rev. Lett. 101, 046101 (2008).

[12] P. Ranitovic, X. M. Tong, C. W. Hogle, X. Zhou, Y. Liu, N. Toshima, M. M. Murnane, and H. C. Kapteyn, Phys. Rev. Lett. 106, 053002 (2011).

[13] X. M. Tong and C. D. Lin, J. Phys. B 38, 2593 (2005).

[14] R. D. Cowan, The Theory of Atomic Structure and Spectra (University of California, Berkeley, 1981).

[15] X. M. Tong and S. I. Chu, Chem. Phys. 217, 119 (1997).

[16] X. M. Tong and S. I. Chu, Phys. Rev. A 55, 3406 (1997).

[17] X. M. Tong, K. Hino, and N. Toshima, Phys. Rev. A 74, 031405 (2006).

[18] X. M. Tong, S. Watahiki, K. Hino, and N. Toshima, Phys. Rev. Lett. 99, 093001 (2007).

[19] M. D. Davidson, J. Wals, H. G. Muller, and H. B. van Linden van den Heuvell, Phys. Rev. Lett. 71, 2192 (1993).

[20] E. S. Toma, H. G. Muller, P. M. Paul, P. Breger, M. Cheret, P. Agostini, C. Le Blanc, G. Mullot, and G. Cheriaux, Phys. Rev. A 62, 061801 (2000).

[21] S. I. Chu and J. Cooper, Phys. Rev. A 32, 2769 (1985). 
[22] R. R. Freeman, P. H. Bucksbaum, H. Milchberg, S. Darack, D. Schumacher, and M. E. Geusic, Phys. Rev. Lett. 59, 1092 (1987).

[23] P. B. Corkum, N. H. Burnett, and F. Brunel, Phys. Rev. Lett. 62, 1259 (1989).

[24] P. Ranitovic et al., New J. Phys. 12, 013008 (2010).
[25] P. Ranitovic, X. M. Tong, C. W. Hogle, X. Zhou, Y. Liu, N. Toshima, M. M. Murnane, and H. C. Kapteyn, Phys. Rev. Lett. 106, 193008 (2011).

[26] H. G. Muller and F. C. Kooiman, Phys. Rev. Lett. 81, 1207 (1998).

[27] O. Guyetand et al., J. Phys. B 41, 065601 (2008). 